\title{
On the Lower Bound of Reconstruction Error for Spectral Filtering Based Privacy Preserving Data Mining
}

\author{
Songtao $\mathrm{Guo}^{1}$, Xintao $\mathrm{Wu}^{1, \star}$, and Yingjiu $\mathrm{Li}^{2}$ \\ ${ }^{1}$ UNC Charlotte \\ ${ }^{2}$ Singapore Management Univ.
}

\begin{abstract}
Additive Randomization has been a primary tool to hide sensitive private information during privacy preserving data mining. The previous work based on Spectral Filtering empirically showed that individual data can be separated from the perturbed one and as a result privacy can be seriously compromised. Our previous work initiated the theoretical study on how the estimation error varies with the noise and gave an upper bound for the Frobenius norm of reconstruction error using matrix perturbation theory. In this paper, we propose one Singular Value Decomposition (SVD) based reconstruction method and derive a lower bound for the reconstruction error. We then prove the equivalence between the Spectral Filtering based approach and the proposed SVD approach and as a result the achieved lower bound can also be considered as the lower bound of the Spectral Filtering based approach.
\end{abstract}

\section{Introduction}

Additive randomization has been a primary tool to hide sensitive private information during privacy preserving data mining. Consider a data set $U$ with $m$ records of $n$ attributes and a noise data set $V$ with same dimensions as $U$. The random value perturbation techniques generate a perturbed data matrix $\tilde{U}=U+V$. Let $\hat{U}$ denote the estimate which the users (or attackers) can achieve. To preserve utility, we mean certain aggregate characteristics (i.e., mean and covariance matrices for numerical data, or marginal totals in contingency table for categorical data) of $U$ should remain basically unchanged in perturbed data $\tilde{U}$ or can be restored from estimated data $\hat{U}$. In other words, distributions of $U$ can be approximately reconstructed from perturbed data $\tilde{U}$ when some a-priori knowledge (e.g., distribution, statistics etc.) about noise $V$ is available using distribution reconstruction approaches (e.g., [2]1]).

To preserve privacy, we mean not only the difference between $\tilde{U}$ and $U$ but also that of between $\hat{U}$ and $U$ should be larger than some tolerated threshold. The authors, in [5] proposed a random matrix-based spectral filtering technique to retrieve original individual data from the perturbed data (a similar Principle Component Analysis based approach investigated in [4]). As a result individual privacy can be seriously compromised.

The previous work only empirically assessed the effects of perturbation on the accuracy of the estimated individual value. Hence one important question is what the

\footnotetext{
* This research was supported by USA National Science Foundation Grant CCR-0310974 and IIS-0546027.
} 
explicit form between reconstruction accuracy and noise added. In our recent work [3], we derived one upper bound for the Frobenius norm of $\hat{U}-U$ in terms of some knowledge of $V$ (e.g., the Frobenius and 2-norm of $V$ ) using the matrix perturbation theory. In this paper, we focus on the lower bound for the Frobenius norm of $\hat{U}-U$. While the upper bound may be exploited by attackers to determine how close their estimates are from the original data, the lower bound can help data owners determine how much noise should be added to satisfy one given threshold of tolerated privacy breach.

Since the traditional matrix perturbation theory [6] mainly focuses on how the eigenvalues and the angle between eigenvectors (or invariance subspaces) of a perturbed matrix $A$ is upper bounded by one perturbation, we cannot borrow any result to derive the lower bound. In this paper, we propose one Singular Value Decomposition (SVD) based reconstruction method and derive a lower bound for the reconstruction error. We then prove the equivalence between the Spectral Filtering based approach and the proposed SVD approach and as a result the achieved lower bound of SVD approach can also be considered as the lower bound of the Spectral Filtering based approach.

\section{Spectral Filtering Reconstruction Method}

We use the tilde conventions to denote perturbations. A symbol with a tilde over it denotes a perturbed quantity. The unperturbed quantity is denoted by the same symbol without a tilde. We denote $A=U^{T} U$ as the covariance matrix of $U$. The eigenvalues of $A, \Lambda(A)=\left\{\lambda_{1}, \cdots, \lambda_{n}\right\}$, and their corresponding eigenvectors $\left[e_{1}, \cdots, e_{n}\right]$ can be obtained, where $\lambda_{1} \geq \lambda_{2} \geq \cdots \geq \lambda_{n}$. And those of $\tilde{A}$ are $\Lambda(\tilde{A})=\left\{\tilde{\lambda}_{1}, \cdots, \tilde{\lambda}_{n}\right\}$.

We cast many of our analysis in terms of absolute and relative errors of matrix norm (i.e., $\|A\|_{F}$ and $\|A\|_{2}$ denote the Frobenius norm and 2-norm respectively), instead of component-wise bounds. The use of absolute and relative errors gives perturbation bounds a simplicity that makes them easier to interpret. Basically, the Frobenius form is used to measure the magnitude of data in total while the 2-norm is used to denote the largest eigenvalue of covariance matrix.

\subsection{Spectral Filtering Revisited}

The authors, in [5], provided an explicit filtering procedure as shown below.

1. Calculate the covariance matrix of $\tilde{U}$ by $\tilde{A}=\tilde{U}^{T} \tilde{U}$.

2. Apply spectral decomposition on $\tilde{A}$ to get $\tilde{A}=\tilde{\tilde{Q}} \tilde{\Lambda} \tilde{Q}^{T}$, where $\tilde{Q}$ is orthogonal matrix whose column vectors are eigenvectors of $\tilde{A}$, and $\tilde{\Lambda}$ is the diagonal matrix with the corresponding eigenvalues on its diagonals.

3. Using random matrix theory, the pair of $\lambda_{V_{\min }}$ and $\lambda_{V_{\max }}$, which provide the theoretical bounds of the eigenvalues corresponding to the matrix $V^{T} V$, are obtained.

4. Extract the first $k$ components of $\tilde{A}$ as the principal components by $k=\max \left\{i \mid \tilde{\lambda}_{i} \geq\right.$ $\left.\lambda_{V_{\max }}\right\} . \tilde{e}_{1}, \tilde{e}_{2}, \cdots, \tilde{e}_{k}$ are the corresponding eigenvectors, which form an orthonormal basis of a subspace $\tilde{\chi}$. Let $\tilde{Q}_{k}=\left[\begin{array}{ll}\tilde{e}_{1} & \tilde{e}_{2} \cdots \tilde{e}_{k}\end{array}\right]$. The orthogonal projection on to $\tilde{\chi}$ is $P_{\tilde{\chi}}=\tilde{Q}_{k} \tilde{Q}_{k}^{T}$

5. Obtain the estimated data set using $\hat{U}=\tilde{U} P_{\tilde{\chi}}$. 
The noise matrix $V$ considered in [5] and this paper is generated using i.i.d. Gaussian distribution with zero mean and known variance. This represents the scenario where the noise is completely independent with original data.

The authors, in [3], investigated the explicit relation between $\hat{U}-U$ and the noise and derived the upper bound of $\|\hat{U}-U\|_{F}$ in terms of $\|V\|_{F}$ shown as

$$
\|\hat{U}-U\|_{F} \leq\|\tilde{U}\|_{F} \frac{2\|V\|_{F}^{2}}{\left(\tilde{\lambda}_{k}-\|E\|_{2}\right)-\sqrt{2}\|V\|_{F}^{2}}+\sqrt{k / n}\|V\|_{F}
$$

where $E=V^{T} V$ is the derived perturbation on covariance matrix $A=U^{T} U$.

\subsection{Strategies of Determining k}

The original Spectral Filtering algorithm applied the following strategy to determine the first $k$ eigen components.

Strategy 1. $k=\max \left\{i \mid \tilde{\lambda}_{i} \gtrsim \lambda_{V_{\max }}\right\}$. When the data set is large, $\lambda_{V_{\max }} \approx \lambda_{V_{\min }} \approx$ $\lambda_{V}$. It becomes: $k=\max \left\{i \mid \tilde{\lambda}_{i} \geq \lambda_{V}\right\}$

We would point out that the previous Strategy 1 applied in [5] in general would not give the optimal reconstruction. The reason is that it aims to include all significant eigen components (with $\lambda_{i}>0$ ) in projection space for reconstruction. However, since the inclusion of one eigen component also brings some additional noise projected on that eigen vector, the benefit due to inclusion of one insignificant eigen component may be diminished by the side effect due to the additional noise projected on this eigen vector.

In this paper, we propose a new strategy (as shown in Strategy 2) which compares the benefit due to inclusion of one component with the loss due to the additional projected noise. We shall show that Strategy 2 is expected to give one approximate optimal reconstruction.

Strategy 2. The estimated data using $\hat{U}=\tilde{U} P_{\tilde{\chi}}=\tilde{U} \tilde{Q}_{k} \tilde{Q}_{k}^{T}$ is approximate optimal when $k=\min \left\{i \mid \tilde{\lambda}_{i}<2 \lambda_{V}\right\}-1$.

Proof. In the Spectral Filtering method, when we select the first $k$ components, the error matrix can be expressed as

$$
\begin{aligned}
f(k) & =\hat{U}-U \\
& =(U+V) \tilde{Q}_{k} \tilde{Q}_{k}^{T}-U \\
& =(U+V) \tilde{Q}\left(\begin{array}{cc}
I_{k} & 0 \\
0 & 0
\end{array}\right) \tilde{Q}^{T}-U \\
& =V \tilde{Q}\left(\begin{array}{rr}
I_{k} & 0 \\
0 & 0
\end{array}\right) \tilde{Q}^{T}-U\left[\tilde{Q} I \tilde{Q}^{T}-\tilde{Q}\left(\begin{array}{rr}
I_{k} & 0 \\
0 & 0
\end{array}\right) \tilde{Q}^{T}\right] \\
& =V \tilde{Q}\left(\begin{array}{rr}
I_{k} & 0 \\
0 & 0
\end{array}\right) \tilde{Q}^{T}-U \tilde{Q}\left(\begin{array}{cc}
0 & 0 \\
0 & I_{n-k}
\end{array}\right) \tilde{Q}^{T}
\end{aligned}
$$

Similarly, when we select the first $k+1$ components, the error matrix becomes

$$
f(k+1)=V \tilde{Q}\left(\begin{array}{rr}
I_{k+1} & 0 \\
0 & 0
\end{array}\right) \tilde{Q}^{T}-U \tilde{Q}\left(\begin{array}{ll}
0 & 0 \\
0 & I_{n-k-1}
\end{array}\right) \tilde{Q}^{T}
$$




$$
\begin{aligned}
& =V\left[\tilde{Q}\left(\begin{array}{cc}
I_{k} & 0 \\
0 & 0
\end{array}\right) \tilde{Q}^{T}+\tilde{e}_{k+1} \tilde{e}_{k+1}^{T}\right]-U\left[\tilde{Q}\left(\begin{array}{cc}
0 & 0 \\
0 & I_{n-k}
\end{array}\right) \tilde{Q}^{T}-\tilde{e}_{k+1} \tilde{e}_{k+1}^{T}\right] \\
& =\left(V \tilde{Q}\left(\begin{array}{cc}
I_{k} & 0 \\
0 & 0
\end{array}\right) \tilde{Q}^{T}-U \tilde{Q}\left(\begin{array}{cc}
0 & 0 \\
0 & I_{n-k}
\end{array}\right) \tilde{Q}^{T}\right)+V \tilde{e}_{k+1} \tilde{e}_{k+1}^{T}+U \tilde{e}_{k+1} \tilde{e}_{k+1}^{T} \\
& =f(k)+V \tilde{e}_{k+1} \tilde{e}_{k+1}^{T}+U \tilde{e}_{k+1} \tilde{e}_{k+1}^{T}
\end{aligned}
$$

The last two parts in Equation 3 are the projections of noise and data on the $(\mathrm{k}+1)$ th eigenvector. Assume $\tilde{e}_{i} \approx e_{i}$, the strength of the data projection can be approximated as

$$
\begin{aligned}
\left\|U \tilde{e}_{k+1} \tilde{e}_{k+1}^{T}\right\|_{F}^{2} & \approx\left\|U e_{k+1} e_{k+1}^{T}\right\|_{F}^{2} \\
& =\operatorname{Tr}\left[\left(U e_{k+1} e_{k+1}^{T}\right)^{T}\left(U e_{k+1} e_{k+1}^{T}\right]\right. \\
& =\operatorname{Tr}\left(e_{k+1} e_{k+1}^{T} U^{T} U e_{k+1} e_{k+1}^{T}\right) \\
& =\operatorname{Tr}\left[e_{k+1} e_{k+1}^{T}\left(\sum_{i=1}^{n} \lambda_{i} e_{i} e_{i}^{T}\right) e_{k+1} e_{k+1}^{T}\right] \\
& =\operatorname{Tr}\left(\lambda_{k+1} e_{k+1} e_{k+1}^{T}\right) \\
& =\lambda_{k+1}
\end{aligned}
$$

For i.i.d noise, the effect of the projection on any vector should be the same. Thus,

$$
\left\|V \tilde{e}_{k+1} \tilde{e}_{k+1}^{T}\right\|_{F}^{2} \approx \lambda_{V}
$$

Hence, we include the i-th component only when $\lambda_{i} \geq \lambda_{V}$. The benefit due to inclusion of the i-th eigen component is larger than the loss due to the noise projected along the $\mathrm{i}$-th eigen component.

It is easy to derive

$$
\begin{gathered}
\operatorname{Cov}\left(U_{i}+V_{i}, U_{j}+V_{j}\right)=\operatorname{Cov}\left(U_{i}, U_{j}\right)+E\left(V_{i} * V_{j}\right) \\
\operatorname{Var}\left(U_{i}+V_{i}\right)=\operatorname{Var}\left(U_{i}\right)+\operatorname{Var}\left(V_{i}\right)
\end{gathered}
$$

when the noise is independent to the data and also has no correlation among the noise.

Since $\tilde{\lambda}_{i}=\lambda_{i}+\lambda_{V} \geq 2 \lambda_{V}$, hence

$$
k=\min \left\{i \mid \tilde{\lambda}_{i}<2 \lambda_{V}\right\}-1
$$

\section{Our SVD-Based Reconstruction}

In this section, we first present one SVD based reconstruction method and then derive the lower bound using the well-known Mirsky Theorem for SVD decomposition. We shall prove the equivalence between the proposed SVD method and Spectral Filtering method in Section 4. Hence the derived lower bound from SVD method can also be considered as the lower bound of Spectral Filtering method. 


\subsection{SVD Reconstruction}

Singular Value Decomposition (SVD) decomposes a matrix $U \in R^{m \times n}$ (say $m \geq n$ ) into the product of two unitary matrices, $L \in R^{m \times m}, R \in R^{n \times n}$, and a pseudo-diagonal matrix $D=\operatorname{diag}\left(\sigma_{1}, \cdots, \sigma_{\rho}\right) \in R^{m \times n}$, such that $U=L D R^{T}$ or $U=\sum_{i=1}^{n} \sigma_{i} l_{i} r_{i}^{T}$. The diagonal elements $\sigma_{i}$ of $D$ are referred to as singular values, which are, by convention, sorted in descending order: $\sigma_{1} \geq \sigma_{2} \geq \cdots \geq \sigma_{n} \geq 0$. The columns $l_{i}$ and $r_{i}$ of $\mathrm{L}$ and R are respectively called the left and right singular vectors of $U$. Similarly let $\tilde{U}=U+V$ be a perturbation of $U$ and let $\tilde{U}=\tilde{L} \tilde{D} \tilde{R}^{T}$ be a perturbation of $\tilde{U}$.

input $\tilde{U}$, a given perturbed data set

$V$, a noise data set

output $\hat{U}$, a reconstructed data

BEGIN

$1 \quad$ Apply SVD on $\tilde{U}$ to get $\tilde{U}=\tilde{L} \tilde{D} \tilde{R}^{T}$

2 Apply SVD on $V$ and assume $\sigma_{V}$ is the largest singular value of $V$

3 Determine the first $k$ components of $\tilde{U}$ by $k=\min \left\{i \mid \tilde{\sigma}_{i}<\sqrt{2} \sigma_{V}\right\}-1$

Assume $\tilde{\sigma}_{1} \geq \tilde{\sigma}_{2} \geq \cdots \tilde{\sigma}_{k}$ and $\tilde{l}_{i}, \tilde{r}_{i}$ are the corresponding left and right singular vectors

$4 \quad$ Reconstructing $U$ approximately as

END

$$
\hat{U}=\tilde{U}_{k}=\sum_{i=1}^{k} \tilde{\sigma}_{i} \tilde{l}_{i} \tilde{r}_{i}^{T}
$$

Fig. 1. SVD Based Reconstruction Algorithm

Figure 1 shows our SVD based reconstruction method. Please note that the strategy used for the SVD based reconstruction is $k=\min \left\{i \mid \tilde{\sigma}_{i}<\sqrt{2} \sigma_{V}\right\}-1$. We shall show its equivalence with the Strategy 2 of the Spectral Filtering method in Section 4.

\subsection{Lower Bound Determination}

Lower Bound. Consider $\hat{U}=\tilde{U}_{k}=\tilde{L}_{k} \tilde{D}_{k} \tilde{R}_{k}^{T}$ as the estimation of the original data set $\mathrm{U}$. The estimation error between $\hat{U}$ and $\mathrm{U}$ has its lower bound:

$$
\|\hat{U}-U\|_{F} \geq\left\|U_{k}-U\right\|_{F}
$$

where $k=\min \left\{i \mid \tilde{\sigma}_{i}<\sqrt{2} \sigma_{V}\right\}-1$.

Proof. $U_{k}$ and $U$ are matrices of the same dimensions with singular values

$$
\begin{aligned}
& \tilde{\sigma}_{1} \geq \tilde{\sigma}_{2} \geq \cdots \geq \tilde{\sigma}_{n} \\
& \sigma_{1} \geq \sigma_{2} \geq \cdots \geq \sigma_{n}
\end{aligned}
$$


Since $\tilde{U}_{k}=\tilde{L}_{k} \tilde{D}_{k} \tilde{R}_{k}^{T}$

$$
\tilde{\sigma}_{k+1}=\cdots=\tilde{\sigma}_{n}=0
$$

By Mirsky's theorem [6]

$$
\|\hat{U}-U\|_{F}^{2} \geq \sum_{i=1}^{n}\left|\tilde{\sigma}_{i}-\sigma_{i}\right|^{2} \geq \sigma_{k+1}^{2}+\cdots+\sigma_{n}^{2}=\left\|U_{k}-U\right\|_{F}^{2}
$$

The relationship between the reconstruction bias and perturbation (especially the lower bound) will, in turn, guide us to add noise into the original data set. The lower bound gives data owners the worst case security assurance since it is bounded any matrix $B$ of rank not greater than $k$ derived by attackers. In order to preserve privacy, data owners need to make sure $\|\hat{U}-U\|_{F} /\|U\|_{F}$ is greater than the privacy threshold $\tau$, specified by users.

Based on the derived lower bound,

$$
\tau\|U\|_{F} \leq\left\|U_{k}-U\right\|_{F}=\sigma_{k+1}^{2}+\cdots \sigma_{n}^{2}
$$

Hence $k$ which might be chosen by attackers can be determined by

$$
k=\max \left\{i \mid \tau \leq\left(\sigma_{i+1}^{2}+\cdots \sigma_{n}^{2}\right) /\|U\|_{F}\right\}
$$

Based on our approximate optimal strategy, $\lambda_{i} \geq \lambda_{V}$, the data owner should add an i.i.d. noise $\mathrm{V}$ and let the eigenvalue of $\left(V^{T} V\right)$ satisfy

$$
\lambda_{k+1}<\lambda_{V} \leq \lambda_{k}
$$

Since $\lambda_{V}$ is the eigenvalue of $V^{T} V$, the variance of the noise can be derived $\operatorname{Var}(V)=$ $\lambda_{V} /(m-1)$, where $m$ is the number of row in $V$.

\section{Equivalence of SVD and SF Reconstruction}

SVD explicitly constructs orthonormal bases for the nullspace and range of a matrix. $U=L D R^{T}$. The non-zero singular values for $U$ are precisely the square roots of the non-zero eigenvalues of the positive semi-definite matrix $U U^{T}$, and these are precisely the square roots of the non-zero eigenvalues of $U^{T} U$. Furthermore, the columns of $L$ are eigenvectors of $U U^{T}$ and the columns of $R$ are eigenvectors of $U^{T} U$.

Theorem 1. The reconstructed data from Spectral Filtering is

$$
\hat{U}_{S F}=\tilde{U} P_{\tilde{\chi}}=\tilde{U} \tilde{Q}_{k} \tilde{Q}_{k}^{T}
$$

where $k=\min \left\{i \mid \tilde{\lambda}_{i}<2 \lambda_{V}\right\}-1$ while the reconstructed data from SVD is

$$
\hat{U}_{S V D}=\tilde{L}_{k} \tilde{D}_{k} \tilde{R}_{k}^{T}
$$

where $k=\min \left\{i \mid \tilde{\sigma}_{i}<\sqrt{2} \sigma_{V}\right\}-1$. We have $\hat{U}_{S F}=\hat{U}_{S V D}$ and the $k$ determined by $k=\min \left\{i \mid \tilde{\lambda}_{i}<2 \lambda_{V}\right\}-1$ and determined by $k=\min \left\{i \mid \tilde{\sigma}_{i}<\sqrt{2} \sigma_{V}\right\}-1$ are the same. 
Proof. We first prove these two methods are equivalent. Since $\tilde{R}_{k}=R\left(\begin{array}{c}I_{k} \\ 0\end{array}\right)$,

$$
\tilde{U} \tilde{R}_{k}=\tilde{U} \tilde{R}\left(\begin{array}{c}
I_{k} \\
0
\end{array}\right)=\left(\tilde{L} \tilde{D} \tilde{R}^{T}\right) \tilde{R}\left(\begin{array}{c}
I_{k} \\
0
\end{array}\right)=\tilde{L} \tilde{D}\left(\begin{array}{c}
I_{k} \\
0
\end{array}\right)=\tilde{L}_{k} \tilde{D}_{k}
$$

Since the columns of right singular vectors $(\tilde{R})$ are the eigenvectors of $\tilde{U}^{T} \tilde{U}$, that is $\tilde{Q}=\tilde{R}$. Then

$$
\hat{U}_{S F}=\tilde{U} \tilde{R}_{k} \tilde{R}_{k}^{T}=\tilde{L}_{k} \tilde{D}_{k} \tilde{R}_{k}^{T}=\hat{U}_{S V D}
$$

We then prove the equivalence of determining $k$. Based on the fact that the singular value of $U$ are the square root of eigenvalues of $U^{T} U$ or $U U^{T}$, we have:

$$
\begin{gathered}
\tilde{\sigma}_{i}=\sqrt{\lambda_{i}\left(\tilde{U}^{T} \tilde{U}\right)}=\sqrt{\tilde{\lambda}_{i}} \\
\sqrt{2} \sigma_{V}=\sqrt{2 \lambda\left(V^{T} V\right)}=\sqrt{2 \lambda_{V}}
\end{gathered}
$$

So, $\tilde{\sigma}_{i}<\sqrt{2} \sigma_{V} \Longleftrightarrow \tilde{\lambda}_{i}<2 \lambda_{V}$. Hence, $\min \left\{i \mid \tilde{\sigma}_{i}<\sqrt{2} \sigma_{V}\right\}-1=\min \left\{i \mid \tilde{\lambda}_{i}<\right.$ $\left.2 \lambda_{V}\right\}-1$.

\section{Empirical Evaluations}

In our experiment, we use the artificial dataset, as specified similarly in [5]. Specifically, $U$ is a highly correlated data set with 35 variables. Each feature has a specific trend like sinusoidal, square, and triangular shape and there is no dependency between any two features. The additive noise follows i.i.d Gaussian distribution $\mathrm{N}(0, \mathrm{COV})$, where covariance matrix $C O V=\operatorname{diag}\left(\sigma^{2}, \cdots, \sigma^{2}\right)$ (The same as in [5]). From the previous discussion, we have $\|V\|_{F} \approx \sqrt{\sigma^{2} m n}$. In our following experiments, we perturb the original data by different level of noises, which are generated by varying the covariance matrix $C O V$. For each perturbed data, we use our SVD technique to reconstruct the point-wise data.

It is easy to see that different $k$ leads to different reconstruction errors (which is measured by $\left.\operatorname{re}(U, \hat{U})=\|U-\hat{U}\|_{F} /\|U\|_{F}\right)$. From Table 1, we can see our SVD method (or the Spectral Filtering method with our Strategy 2) can achieve optimal results for all perturbations. Note the values with * denote the results achieved by our algorithm while the values in with $\dagger$ denote the results following the previous Strategy 1 . The values in bold font highlight the best results achieved by varying $k$. Our proposed SVD method (or the Spectral Filtering method with our Strategy 2) can match the best results while the Spectral Filtering with the previous Strategy 1 suffers when relative large perturbations are introduced.

When we examine the original data, there exist 6 principle components as the data is highly correlated among 35 features. Hence, for relative small perturbations, the effects on the remaining 29 components are safely filtered. However, when we increase the noise level (i.e., $\|V\|_{F}$ increases), the noise will tend to affect the determination of $k$. This is because the gain of correct inclusion of some (not very significant) principal component is diminished by the loss due to the inclusion of noise projected on that component. 
Table 1. The relative error $r e(U, \hat{U})$ vs. varying V. The values with $*$ denote the results following Strategy 2 , while the values with $\dagger$ denote the results following the Strategy 1 . The bold values indicate those best estimations achieved by the spectral filtering technique. The noise is i.i.d. Gaussian noise with zero mean and equal variance on each dimensions.

\begin{tabular}{|c|c|c|c|c|c|c|c|c|c|c|}
\hline \multicolumn{2}{|c|}{ noise } & V1 & V2 & V3 & V4 & V5 & V6 & V7 & V8 & V9 \\
\hline \multicolumn{2}{|c|}{ variance } & 0.213 & 0.333 & 0.491 & $\overline{0.750}$ & 1.007 & 1.524 & 2.040 & 2.430 & $\overline{4.81}$ \\
\hline $\mid V\left\|_{F} /\right\|$ & $\bar{U} \|_{F}$ & 0.628 & 0.786 & 0.954 & 1.178 & 1.366 & 1.677 & 1.944 & 2.121 & 2.985 \\
\hline \multirow{7}{*}{$\operatorname{re}(U, \hat{U})$} & $\mathrm{k}=1$ & 0.821 & 0.825 & 0.830 & 0.839 & 0.847 & 0.863 & 0.877 & 0.890 & 0.960 \\
\hline & $\mathrm{k}=2$ & 0.649 & 0.659 & 0.671 & 0.692 & 0.711 & 0.750 & 0.783 & 0.810 & $* 0.956$ \\
\hline & $\mathrm{k}=3$ & 0.440 & 0.461 & 0.488 & 0.529 & 0.565 & 0.636 & 0.694 & (0.739 & 0.964 \\
\hline & $\mathrm{k}=4$ & 0.297 & 0.337 & $* 0.383$ & $* 0.450$ & $* 0.506$ & *0.607 & *0.687 & 0.748 & 1.032 \\
\hline & $\mathrm{k}=5$ & 0.271 & $* 0.324$ & 0.383 & 0.465 & 0.532 & 0.651 & 0.745 & 0.816 & 1.141 \\
\hline & $\mathrm{k}=6$ & 0.260 & $\dagger 0.325$ & $\dagger 0.395$ & $\dagger 0.489$ & $\dagger 0.567$ & $\dagger 0.699$ & $\dagger 0.805$ & $\dagger 0.883$ & $\dagger 1.245$ \\
\hline & $\mathrm{k}=7$ & 0.282 & 0.353 & 0.428 & 0.530 & 0.614 & 0.757 & 0.873 & 0.956 & 1.348 \\
\hline
\end{tabular}

\section{Conclusion}

Spectral filtering based technique has recently been investigated as a major means of point-wise data reconstruction [5|4]. It was empirically shown that under certain conditions this technique may be exploited by attackers to breach the privacy protection offered by randomization based privacy preserving data mining methods. We present an explicit lower bound of reconstruction accuracy in terms of Frobenius norm. This lower bound can help users determine how much and what kind of noise should be added when one tolerated privacy breach threshold is given. In the future we are interested in deriving the bounds at point-wise level.

\section{References}

1. D. Agrawal and C. Agrawal. On the design and quantification of privacy preserving data mining algorithms. In Proceedings of the 20th Symposium on Principles of Database Systems, 2001 .

2. R. Agrawal and R. Srikant. Privacy-preserving data mining. In Proceedings of the ACM SIGMOD International Conference on Management of Data, pages 439-450. Dallas, Texas, May 2000.

3. S. Guo and X. Wu. On the use of spectral filtering for privacy preserving data mining. In Proceedings of the 21st ACM Symposium on Applied Computing, pages 622-626, April 2006.

4. Z. Huang, W. Du, and B. Chen. Deriving private information from randomized data. In Proceedings of the ACM SIGMOD Conference on Management of Data. Baltimore, MA, 2005.

5. H. Kargupta, S. Datta, Q. Wang, and K. Sivakumar. On the privacy preserving properties of random data perturbation techniques. In Proceedings of the 3rd IEEE International Conference on Data Mining, pages 99-106, 2003.

6. G. Stewart and J. Sun. Matrix Perturbation Theory. Academic Press, 1990. 